\title{
Corticostriatopallidal Neuroprotection by Adenovirus-Mediated Ciliary Neurotrophic Factor Gene Transfer in a Rat Model of Progressive Striatal Degeneration
}

\author{
Vincent Mittoux, ${ }^{1 *}$ Stéphane Ouary, ${ }^{1 *}$ Christelle Monville, ${ }^{2}$ Fabrice Lisovoski, ${ }^{2}$ Thomas Poyot, ${ }^{1}$ \\ Françoise Condé, ${ }^{1}$ Carole Escartin, ${ }^{1}$ Régine Robichon, ${ }^{2}$ Emmanuel Brouillet, ${ }^{1}$ Marc Peschanski, ${ }^{2}$ and \\ Philippe Hantraye ${ }^{1,3}$ \\ 1 Unité de Recherche Associée 2210, Commissariat à l'Energie Atomique, Centre National de la Recherche Scientifique, \\ Service Hospitalier Frédéric Joliot, 91401 Orsay Cedex, France, 2Institut National de la Santé et de la Recherche Médicale \\ U421, Institut Mondor de Médecine Moléculaire, Faculté de Médecine, 94010 Créteil Cedex, France, and ${ }^{3}$ Unité \\ d'Imagerie Isotopique, Biochimique, et Pharmacologique, Service Hospitalier Frédéric Joliot, Département de la \\ Recherche Médicale, Commissariat à l'Energie Atomique, 91401 Orsay Cedex, France
}

Ciliary neurotrophic factor (CNTF) is a potent protective factor for striatal neurons in animal models of Huntington's disease (HD). Clinical application of this potential therapeutic still requires the design and optimization of delivery systems. In the case of HD, spatial spread in the vast volume occupied by the striatum and long-term delivery of the factor are particular challenges for these systems. We explored the potential of adenovirus-mediated gene transfer to fulfill these requirements by studying the functional and anatomical effects of single-site striatal delivery of CNTF recombinant vectors in a rat model of HD. In an initial series of experiments, unilateral injections of CNTF adenovirus were performed in rats 10, 30, or $90 \mathrm{~d}$ before a $5 \mathrm{~d}$ neurotoxic treatment with systemic 3-nitropropionic acid (3NP). Preservation of striatal neurons was observed at all time points, demonstrating temporally extended neuroprotective effects of the CNTF adenovirus. In a second series of experi- ments, bilateral injections of CNTF adenovirus were performed in the medial aspect of the striatum $10 \mathrm{~d}$ before starting 3NP intoxication. Despite placement of the CNTF-producing vector outside the lateral striatal area susceptible to lesion, massive protection of corticostriatopallidal circuits was observed, associated with significant behavioral benefits. This spatial spread of neuroprotection is discussed with reference to the retrograde transport of the adenovirus vector and the anterograde transport of the transgenic CNTF. Overall, adenovirus-mediated CNTF gene transfer appears to be a potentially useful delivery system for widespread, long-term circuit neuroprotection in HD patients.

Key words: adenovirus vector; ciliary neurotrophic factor; 3-nitropropionic acid; corticostriatopallidal degeneration; neuroprotection; rat; stereology; Huntington's disease
Protection of GABAergic striatal neurons by ciliary neurotrophic factor $(\mathrm{CNTF})$ is a promising prospect for slowing down the pathologic process of Huntington's disease (HD). A number of studies have indeed demonstrated that intracerebral administration of CNTF using either minipumps (Anderson et al., 1996) or encapsulated genetically engineered cells (Emerich et al., 1996; Mittoux et al., 2000) significantly decreases the extent of striatal

\footnotetext{
Received July 25, 2001; revised March 20, 2002; accepted March 20, 2002.

This work was supported by Centre National pour la Recherche Scientifique, Commissariat à l'Energie Atomique (CEA), Institut National de la Santé et de la Recherche Médicale, Association Française contre les Myopathies (AFM), and the European Union (QLRT 1999-00702). V.M. and S.O. are CEA fellows and C.M. is an AFM fellow. We gratefully acknowledge help from Drs. S. Akli, G. Haase, A. Kahn, M. Sendtner, and H. Thoenen in the preparation of the AdRSV.CNTF vector and thank Drs. J. M. Hermel and K. L. Moya for critical reading and comments on this manuscript.

*V.M. and S.O. contributed equally to this work.

Correspondence should be addressed to Dr. Philippe Hantraye, Unité de Recherche Associée 2210, Commissariat à l'Energie Atomique, Centre National de la Recherche Scientifique, Service Hospitalier Frédéric Joliot, 4 place du Général Leclerc, 91401 Orsay Cedex, France. E-mail: hantraye@shfj.cea.fr.

V. Mittoux's present address: ALCIMED Biotechnology, 57 boulevard de Montmorency, 75016 Paris, France.

F. Lisovoski's present address: Centre du Rachis, Clinique Ambroise Paré, 27 boulevard Victor Hugo, 92200 Neuilly-sur-Seine, France.

T. Poyot's present address: Institut de Recherche Internationale SERVIER, 6 place des Pleiades, 92415 Courbevoie cedex, France.

Copyright (C) 2002 Society for Neuroscience $0270-6474 / 02 / 224478-09 \$ 15.00 / 0$
}

cell loss produced by a variety of neurotoxins in rats or nonhuman primates. In vitro experiments have also confirmed a protective effect of CNTF on cells genetically engineered to express the mutated form of the huntingtin protein (Saudou et al., 1998).

Finding an efficient way to deliver CNTF to striatal neurons in patients remains unresolved. Systemic administration of CNTF is not appropriate, because it practically does not cross the bloodbrain barrier and induces major side effects and likely, an immune reaction. Therefore direct intracerebral delivery using in vivo gene therapy constitutes an interesting alternative approach. Adenoviruses are among the most efficient vectors to transfer genes into the brain (Akli et al., 1993; Bajocchi et al., 1993; Davidson et al., 1993; Le Gal La Salle et al., 1993), but their clinical relevance has often been questioned, primarily because of three potential drawbacks: toxicity (Akli et al., 1993; Caillaud et al., 1993; Byrnes et al., 1995), lack of spatial dispersion after intraparenchymal administration (Lisovoski et al., 1997; Peltekian et al., 1997), and suspected decrease in gene expression over time.

Regarding cytotoxicity, a concurrent decrease in toxicity and increase in transgene expression has been obtained recently using a low $\left(\sim 10^{8} \mathrm{pfu}\right)$ titer of vector (Bohn et al., 1999). With regard to the second point, the lack of dispersion of the adenoviral particles at the injection site may be partly compensated for by intrinsic properties of adenoviral vectors such as retrograde trans- 
port of the transgene to the cell bodies and anterograde transport of the transgene product to nerve terminals including axonal collaterals (Akli et al., 1993; Kuo et al., 1995; Lisovoski et al., 1997; Terashima et al., 1997). Interestingly, such spreading of the transgene has been shown previously to trigger astrocyte differentiation in regions located in the projection zones of retrogradely transduced neurons, after intrastriatal injection of CNTF recombinant adenovirus vectors (Lisovoski et al., 1997).

In this context, we aimed to further explore the neuroprotective potential of a CNTF recombinant adenovirus vector in a rat model of striatal neurodegeneration (Ouary et al., 2000). In this model, as in HD, the cell loss is primarily observed in the striatum, but at later times it also extends to other cerebral areas anatomically connected to the caudate-putamen complex, such as various upstream cortical areas and the downstream globus pallidus. The present work investigated whether the use of a singlesite striatal administration of low-titer CNTF adenoviral vector would confer widespread and long-lasting corticostriatopallidal neuroprotection in a chronic lesion rat model of HD.

\section{MATERIALS AND METHODS}

Experimental design. Two separate experiments were performed to examine, respectively, the temporal and the spatial extent of the neuroprotective effects of a single intrastriatal administration of $5 \times 10^{8} \mathrm{pfu}$ of a CNTF recombinant adenovirus vector.

In the first set of experiments, we studied the time course of the neuroprotective effect of CNTF. Adult Lewis rats were unilaterally infected in one striatum with either a CNTF (AdRSV.CNTF) or a control LacZ (AdRSV.LacZ) recombinant adenovirus vector. The survival of striatal neurons was challenged either 10,30 , or $90 \mathrm{~d}$ later by systemically administering 3-nitropropionic acid (3NP), as described previously (Ouary et al., 2000). Animals were killed at the end of $5 \mathrm{~d}$ of neurotoxic treatment for histological assessment and quantification of striatal lesion volumes. In the second set of experiments, we quantified the spatial extent of neuroprotection and the behavioral benefits produced by a single-site, bilateral administration of the CNTF vector into the medial striatum of adult Lewis rats, challenged $10 \mathrm{~d}$ later by systemic $3 \mathrm{NP}$. At the end of the $3 \mathrm{NP}$ treatment, the animals were allowed to recover for $16 \mathrm{~d}$. They were then tested for locomotion activity before killing and stereological neuronal quantification.

$3 N P$ intoxication. A stock solution of 3NP (Fluka, Saint Quentin Fallavier, France) was made fresh at a concentration of $307-316 \mathrm{mg} / \mathrm{ml}$ in deionized water and adjusted to a $\mathrm{pH}$ of 7.4 with $1 \mathrm{~m} \mathrm{NaOH}$ solution, keeping the solution below $+25^{\circ} \mathrm{C}$. The concentration of the stock solution was calculated according to the weight of the heaviest rat per group. Osmotic minipumps (2ML4 model; Alzet Corporation, Palo Alto, CA) were loaded to deliver $60 \mu \mathrm{l}$ per $24 \mathrm{hr}$ for $5 \mathrm{~d}$. The $3 \mathrm{NP}$ concentration in the pump was adjusted to the exact weight of each animal at the day of implantation, so that each rat received a dose of 38 $\mathrm{mg} \cdot \mathrm{kg}^{-1} \cdot \mathrm{d}^{-1}$. The pumps were implanted subcutaneously in the back under ketamine-xylazine anesthesia. This intoxication regimen produces consistent, bilateral striatal degeneration in Lewis rats (Ouary et al., 2000).

Adenovirus vectors. The adenovirus vectors recombinant for genes encoding either the rat $C N T F$ gene and an attached sequence encoding mouse NGF signal peptide to ensure secretion (AdRSV.CNTF) or the reporter gene $\beta$-galactosidase (AdRSV.LacZ), under the control of the long-terminal repeat promoter of the Rous sarcoma virus, have been described previously (Lisovoski et al., 1997). Both adenovirus vectors were obtained at titers up to $10^{12} \mathrm{pfu} / \mathrm{ml}$.

The AdRSV.CNTF vector has previously demonstrated its biological activity in vitro and in vivo (Lisovoski et al., 1997; Haase et al., 1999). To assess the stability and long-term transgene expression of this vector, mRNA from the striatum of two AdRSV.CNTF-injected rats was isolated at 3 months after infection by the Trizol method (Invitrogen, Cergy, France) and a PCR was performed with cDNA derived from $2 \mu \mathrm{g}$ of RNA, $2.5 \mathrm{U}$ of AmpliTaq Polymerase, and a reaction kit (Superscript preamplification system; Invitrogen), in a final volume of $50 \mu \mathrm{l}$. Each cycle of PCR included $30 \mathrm{sec}$ of denaturation at $95^{\circ} \mathrm{C}, 30 \mathrm{sec}$ of primer annealing at $55^{\circ} \mathrm{C}$, and $1 \mathrm{~min}$ of extension/synthesis at $72^{\circ} \mathrm{C}$. One cycle of $72^{\circ} \mathrm{C}$ for $10 \mathrm{~min}$ was performed at the end of the 35 cycles. Primers corresponded to pre-NGF (5'-AGCTCACCTCAGTGTCTGGG) and CNTF exon 2 (5'-ACCATCCACTGAGTCAAGGC), therefore excluding endogenous CNTF mRNA from the analysis (Haase et al., 1999). Each primer was added at $0.2 \mu \mathrm{M}$ per reaction.

As an additional control for a potential neuroprotective effect by the inflammatory response triggered by adenovirus vectors, we evaluated the long-term effects (50 d after infection) of the noncytotoxic titer of LacZ recombinant vector. We also examined the effects of a 10 -fold higher titer of the same vector, in the same volume. Such a nonspecific neuroprotective effect was definitively ruled out because there were no statistical differences in the size of striatal lesions between the infected and noninfected striatum of rats receiving an intrastriatal injection of either PBS $(n=9), 5 \times 10^{8}$ pfu AdRSV.LacZ $(n=8)$, or $5 \times 10^{9}$ pfu $\operatorname{AdRSV.LacZ}(n=6)$, or between striatal lesions in the each of the three experimental groups.

Surgery. Male adult Lewis rats (IFFA Credo, Saint-Germain sur L'Arbresle, France), 12 weeks of age and weighing 300-350 gm (at time of infection), were used in these studies. The animals were housed in groups of three to four in a temperature- and humidity-controlled room that was maintained on a $12 \mathrm{hr}$ light/dark cycle. Food and water were available ad libitum throughout the experiment. Experimentation was performed in strict accordance with the recommendations of the European Ethical Committee (EEC) (86/609 EEC), and French National Ethical Committee (87/848) for care and use of laboratory animals.

Immediately before surgery, rats were anesthetized with a mixture of ketamine-xylazine (mixture of $15 \mathrm{mg} / \mathrm{kg}$ and $3 \mathrm{mg} / \mathrm{kg}$, respectively) and positioned in a Kopf stereotaxic instrument (David Kopf Instruments, Les Ulis, France). A midline incision was made in the scalp, and holes were drilled for the placement of a $5 \mu \mathrm{l}$ Hamilton syringe equipped with a 28 gauge needle. Rats received a unilateral $(n=5-10$ in each group; experiment 1$)$ or bilateral $(n=8-9$ in each group; experiment 2$)$ striatal injection of $5 \times 10^{8} \mathrm{pfu}$ (adjusted to a final volume of $1 \mu \mathrm{l}$ of PBS) of either of the two vectors. The stereotaxic coordinates for striatal infection were: $0.5 \mathrm{~mm}$ anterior to Bregma, $3 \mathrm{~mm}$ lateral from the sagittal suture, and $4.5 \mathrm{~mm}$ below the dura. These coordinates were selected to reach the medial third of the striatum (Paxinos and Watson, 1986), so that the infected/transgene-expressing cells would be located outside the dorsolateral striatal area that exhibits the maximal neuronal loss after 3NP treatment (Ouary et al., 2000). After infection, the skin was sutured closed and the animals were allowed to recover in their home cage. At 10, 30 , or $90 \mathrm{~d}$ after unilateral infection (experiment 1 ) or $10 \mathrm{~d}$ after bilateral infection (experiment 2), all animals were reanesthetized for implantation of the 3NP-releasing minipumps.

Control animals (experiment 1 ) received one unilateral intrastriatal injection of $1 \mu \mathrm{l}$ of PBS $(n=6)$ and AdRSV.LacZ at a concentration of $5 \times 10^{8}(n=8) 10 \mathrm{~d}$ before implantation of the 3 NP-releasing minipumps. For experiment 2, 10 animals were implanted with empty osmotic minipumps and used as sham-operated controls for the behavioral and stereological cell count analyses.

Motor index (experiment 2). Starting on day 1 of the $3 \mathrm{NP}$ intoxication until day 13, animals were evaluated daily for motor impairment. Animals were rated for the presence or absence of a variety of motor abnormalities using a rating scale previously designed in our laboratory (Ouary et al., 2000). As shown in Table 1, this rating scale considers five different criteria associated with motor function. A score of 0 is defined as normal, whereas a maximal score of 8 corresponds to a severely affected animal displaying near-death recumbency.

At $16 \mathrm{~d}$ after removal of the minipumps, motor performances were also assessed quantitatively in the elevated board test, using a video-based motion tracking and analysis system, as described previously (Guyot et al., 1997). Briefly, animals were trained for $5 \mathrm{~d}$ to run across an elevated board. On the fifth day of training, all sham-operated animals were able to cross the board without rearing or stopping, and a test run was recorded for all groups of rats using a video recorder (VM-2900ES; Hitachi, Tokyo, Japan), located laterally to the board. Images were analyzed off-line. The instantaneous position of each animal's geometric center was determined in two dimensions (X-Y position) with a 3.9 $\mathrm{mm} /$ pixel spatial resolution and a $40 \mathrm{msec}$ time resolution. Maximum speed [peak tangential velocity (PTV)] and average tangential velocity (ATV) were then calculated. Mean step size was also determined.

Histology. Animals were killed either at the end of the fifth day of the neurotoxic treatment (experiment 1 ) or at $21 \mathrm{~d}$, after behavioral testing (experiment 2). All animals received an overdose of pentobarbital (120 $\mathrm{mg} / \mathrm{kg}$, i.p.; Sanofi, Libourne, France) and were perfused transcardially with $100 \mathrm{ml}$ of $0.1 \mathrm{M}$ PBS, $\mathrm{pH} 7.4$, followed by $250 \mathrm{ml}$ of $4 \%$ parafor- 


\begin{tabular}{|c|c|}
\hline $\begin{array}{l}\text { Table 1. Clinical rating scale used to quantify } 3 \mathrm{NP} \\
\text { symptoms in rats }\end{array}$ & uced motor \\
\hline Score & $\begin{array}{l}\text { Maximum score } \\
\text { (near-death rat) }\end{array}$ \\
\hline
\end{tabular}

Recumbency

All four legs active and four paws facing the floor

Two hind legs unable to sustain walk and body weight but forepaw able to keep upright position balance

Animal lying on its side, falling when put on its feet, showing rapid breathing, uncoordinated movements and chewing, almost complete paralysis

Hind legs

Normal, active gait

Presence of intermittent dystonia in one leg

Intermittent dystonia in two hind legs or permanent dystonia in one hind leg

Permanent dystonia in two hind legs

Gait

Normal gait

Presence of wobbling, uncoordinated, jumpy gait

\section{Balance}

Ability to keep balance for $10 \mathrm{sec}$ on a $5 \times 10 \mathrm{~cm}$ platform $(15 \mathrm{~cm}$ height)

Inability to keep balance

Grasping

Ability to grasp a cage grid with at least one forepaw for $10 \mathrm{sec}$ Inability to grasp Total

\begin{tabular}{|c|c|}
\hline 0 & - \\
\hline 1 & - \\
\hline 2 & 2 \\
\hline 0 & - \\
\hline 1 & - \\
\hline 2 & - \\
\hline 3 & 3 \\
\hline 0 & - \\
\hline 1 & 1 \\
\hline 0 & - \\
\hline 1 & 1 \\
\hline 0 & - \\
\hline 1 & 1 \\
\hline & 8 \\
\hline
\end{tabular}

maldehyde in $0.1 \mathrm{M}$ phosphate buffer. Brains were removed, postfixed overnight at $4{ }^{\circ} \mathrm{C}$ in the same fixative, and cryoprotected in a $30 \%$ sucrose solution. Coronal brain sections ( $40 \mu \mathrm{m}$ thick) were cut on a freezing microtome and collected in an anatomical series; every 12th section was stained with $0.15 \%$ gallocyanine (Gurr, Poole, UK). Remaining sections were cryoprotected and stored at $-20^{\circ} \mathrm{C}$.

Immunocytochemical experiments were performed using a mouse antivertebrate neuron-specific nuclear protein (NeuN; dilution 1:10,000; Chemicon, Temecula, CA) or rabbit anti- $\beta$-galactosidase (dilution 1:2000; Chemicon). Brain sections were preincubated in PBS containing $5 \%$ normal goat serum (NGS) and $0.3 \%$ Triton $\mathrm{X}-100$ for $30 \mathrm{~min}$ at room temperature. Sections were incubated for $48 \mathrm{hr}$ at room temperature in PBS containing $3.5 \%$ NGS, $0.3 \%$ Triton $\mathrm{X}-100,0.5 \%$ bovine serum albumin, $0.05 \%$ sodium azide, and the primary antibody. Sections were then processed by the avidin-biotin peroxidase method using Vectastain avidin-biotin complex and VIP kits (Vector Laboratories, Burlingame, CA).

Fluoro-Jade (Histo-Chem, Jefferson, AR), a fluorochrome that stains degenerating neurons (Schmued et al., 1997), was used to document the striatal lesion. Briefly, $40-\mu \mathrm{m}$-thick sections were mounted, dried, and immersed in $100 \%$ ethanol, followed by $70 \%$ ethanol and distilled water. Sections were then treated with $0.06 \%$ potassium permanganate for 15 min. After rinsing, sections were immersed in Fluoro-Jade $(0.001 \%$ Fluoro-Jade in $0.1 \%$ acetic acid) for $30 \mathrm{~min}$, rinsed, and rapidly air-dried on a slide warmer.

Quantitative assessment of striatal lesions. The extent of the striatal lesion produced by $3 \mathrm{NP}$ was determined in all animals of experiment 1
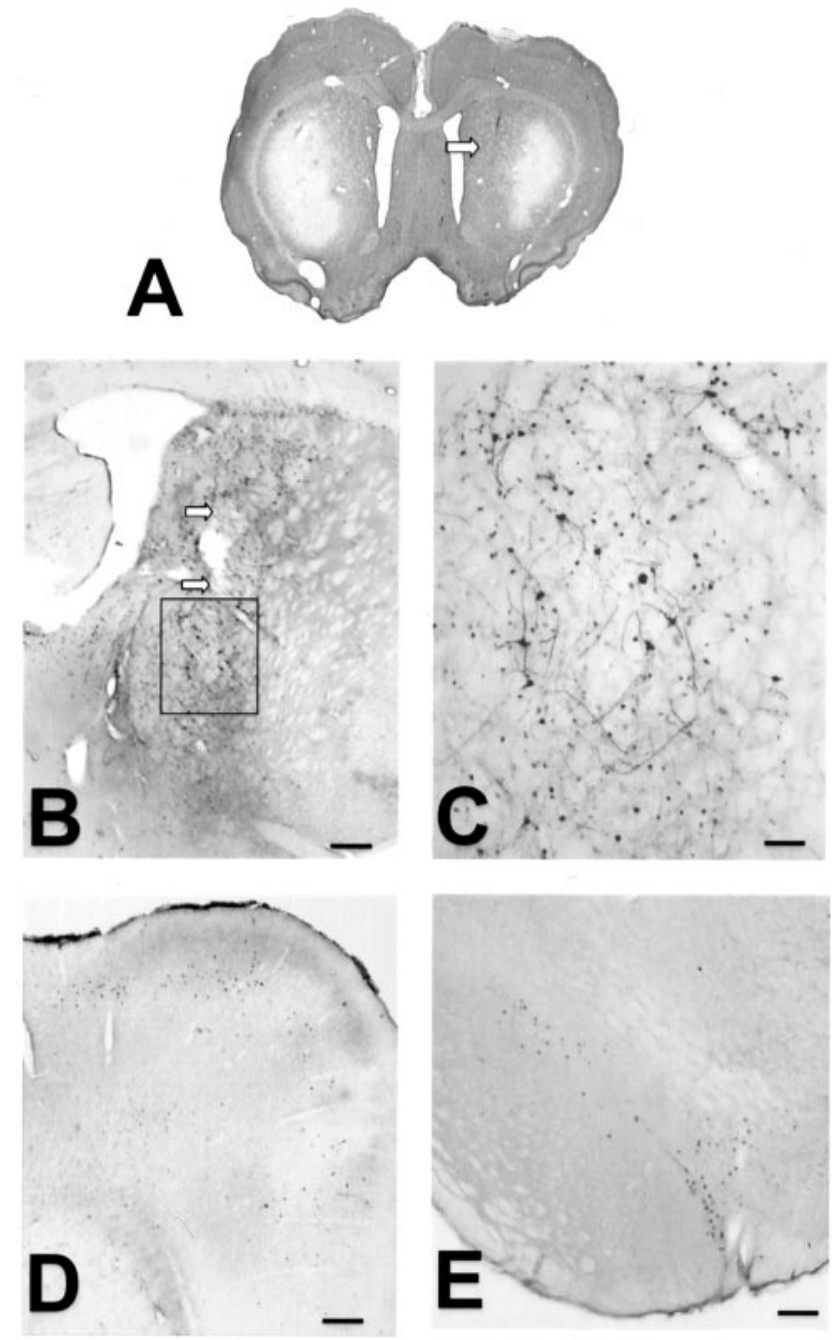

Figure 1. Microphotographs of one intrastriatal injection of AdRSV.LacZ adenovirus in a 3NP-intoxicated rat (experiment 1 , group $L a c Z 10$ ). A, Gallocyanine-stained section showing the bilateral cell loss induced by the $3 \mathrm{NP}$ intoxication and its location in the lateral part of the striatum. The injection needle track (arrow) is located outside the area of cell loss. $B, C$, Section immunostained for $\beta$-galactosidase showing the distribution of immunoreactive cells along the injection needle track (arrows, $B$ ). C (corresponding to the inset in $B$ ), Note that not only the cell nuclei but also some cell processes express $\beta$-galactosidase. $D, E$, Distribution of neurons expressing $\beta$-galactosidase in the medial agranular cortex $(D)$ and in the substantia nigra $(E)$. The distribution of immunoreactive cell bodies corresponds to the injection of AdRSV.LacZ adenovirus shown in $B$. Scale bars: $B, D, E$, $250 \mu \mathrm{m} ; C, 60 \mu \mathrm{m}$.

using a semiautomated image-analysis system. Six to seven equidistant $(200 \mu \mathrm{m})$ gallocyanine-stained sections, selected to obtain a complete rostrocaudal sampling of the striatum, were digitized using a computerassisted morphometry system consisting of an Olympus (Rungis, France) AX70 photo-microscope and a Sony (Tokyo, Japan) HAD Power 3 CCD color video camera connected to an Olympus Pentium II computer equipped with the morphometry software Analysis Pro 3.0. The border of the lesion was traced bilaterally at $1.5 \times$ magnification on each brain section, and the respective lesion volumes were calculated according to the method of Cavalieri.

In experiment 2, stereological neuronal counts were performed in NeuN-immunostained sections from sham-operated animals $(n=9)$, AdRSV.CNTF-injected animals $(n=8)$ and AdRSV.LacZ-injected animals $(n=8)$, killed $16 \mathrm{~d}$ after removal of the osmotic minipumps. Under these conditions, the striatal area of degeneration observed on day 5 of the 3NP intoxication had disappeared, leaving a small scar detectable in 


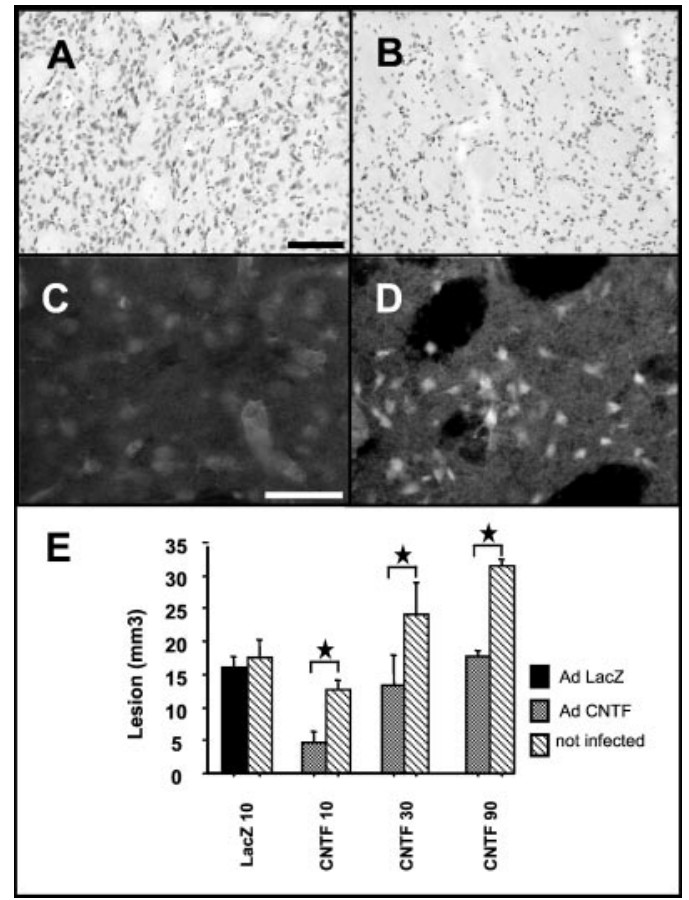

Figure 2. Effects of unilateral striatal injections of adenovirus on the volume of the striatal lesion induced by $3 \mathrm{NP}$ intoxication (experiment 1 ). $A-D$, Photomicrographs of neurons identified by immunostaining for NeuN $(A, B)$ or by Fluoro-Jade staining $(C, D)$ in the lateral striatum of a normal $(A-C)$ and a 3 NP-intoxicated $(B-D)$ animal. In $B$, note the shrinkage and the loss observed in the neuronal population. This neuronal loss is supported by the Fluoro-Jade staining of degenerative neurons (D). E, Quantification of the volume of the 3NP-induced striatal lesion confirms that the unilateral striatal injection of AdRSV.CNTF adenovirus is associated with a reduction of the lesion in the injected striatum, when the adenovirus has been injected 30 and $90 \mathrm{~d}$ as well as $10 \mathrm{~d}$ before the beginning of the $3 \mathrm{NP}$ intoxication. Moreover, there is no statistical difference between the lesion volumes obtained in the striatum of the AdRSV.LacZ-injected group compared with the noninjected contralateral striatum of the AdRSV.LacZ- and the AdRSV.CNTF-injected groups when the adenovirus was injected $10 \mathrm{~d}$ days before the beginning of the 3 NP intoxication. $\star p<0.01$; unpaired $t$ test. Scale bar: $A, B, 100$ $\mu \mathrm{m} ; C, D, 50 \mu \mathrm{m}$.

the lateral part of the striatum, suggesting a spatial reorganization of the surviving neurons and subsequent atrophy of the structure. For each animal, the sections used for neuron counting covered the entire striatum, pallidum, or rostral part of the medial agranular cortex in their entire rostrocaudal length. On average, this corresponded to 11, 11, and 5 sections in the striatum, the pallidum, and the cortex, respectively, with an interspace between adjacent sections of 440, 240, and $240 \mu \mathrm{m}$, respectively. Stereological countings were performed with C.A.S.T.-grid software (Olympus, Albertslund, Denmark). The optical disector (West and Gundersen, 1990) was used to obtain unbiased estimates of total numbers of NeuN-immunoreactive neurons for each structure.

Data analyses. Data were analyzed using Statview (SAS Institute, Inc., Cary, NC; 1991). Statistical analysis was performed using paired or unpaired $t$ tests for lesion volume comparisons, a Mann-Whitney $U$ nonparametric test in the case of the motor impairment index, and a factorial ANOVA followed by a post hoc Scheffe's $F$ test in all other cases. Data are presented as mean \pm SEM.

\section{RESULTS}

All animals recovered uneventfully from administration of adenovirus vectors and showed no behavioral side effects at any time. The injection sites for the adenovirus vectors were located in the medial third of the striatum, outside of the dorsolateral striatal area of 3NP-induced neuronal loss (Fig.

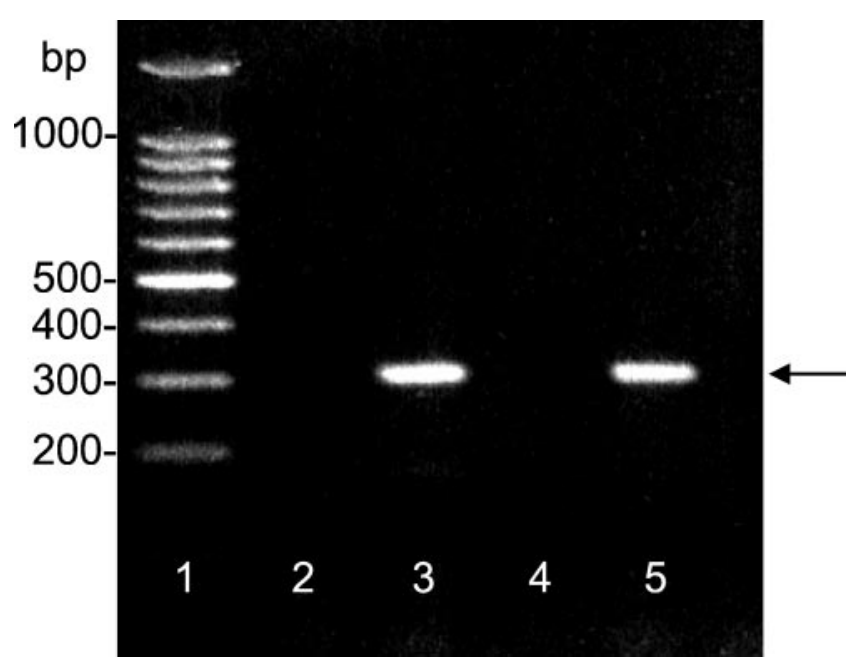

Figure 3. Expression of transgenic mRNA for CNTF in the striatum $90 \mathrm{~d}$ after one injection of $5 \times 10^{8}$ AdRSV.LacZ adenovirus into the right striatum of two rats. mRNA from the ipsilateral (lanes 3 and 5) and contralateral (lanes 2 and 4 ) striatum was reverse transcribed and amplified by PCR. Transgenic CNTF was only found in the injected striatum, at the predicted base pair (bp) size (arrow, lanes 2 and 4). Lane 1, DNA/RNA molecular weight base pair standards.

$1 A)$. In the injected striatum, both cells and fibers were immunoreactive for $\beta$-galactosidase in a cylinder of a few hundred micrometers in diameter around the needle track (Fig. 1B). Immunostaining with $\beta$-galactosidase also confirmed the ability of the AdRSV.LacZ to transfer the LacZ gene not only to striatal cells (Figs. $1 B, C$ ) but also to neurons located in various striatal afferent regions such as the medial agranular cortex (Fig. 1D) and the substantia nigra (Fig. $1 E$ ).

\section{Temporal features of striatal neuroprotective effects provided by AdRSV.CNTF (experiment 1)}

Intoxication of rats by $3 \mathrm{NP}$ induced a striatal-selective lesion, located in the lateral part of the structure (Fig. $1 A$ ). In agreement with previous findings (Beal et al., 1993; Guyot et al., 1997; Ouary et al., 2000), the lesion was characterized by a severe loss of NeuN-immunopositive neurons (Fig. $2 A, B$ ) and intense FluoroJade staining within the lesion area (Fig. $2 D$ ), as opposed to the complete absence of staining in the normal brain (Fig. 2C).

In rats injected in the left striatum with AdRSV.LacZ $10 \mathrm{~d}$ before initiation of the 3NP intoxication ( $\operatorname{LacZ} 10, n=6$ ) (Fig. $2 E)$, the mean volume of the striatal lesion was $16.1 \pm 1.6$ and $17.6 \pm 2.6 \mathrm{~mm}^{3}$ in the left and right striatum, respectively, with no statistical difference between the injected and noninjected sides. In rats injected in the left striatum with AdRSV.CNTF $10 \mathrm{~d}$ before initiation of the 3NP intoxication (Fig. $2 E)(n=9, C N T F 10)$, a $64 \%$ reduction in lesion volume was observed compared with the noninfected contralateral side $\left(4.57 \pm 1.86\right.$ vs $12.71 \pm 1.46 \mathrm{~mm}^{3}$; $p<0.001)$. Waiting 30 or $90 \mathrm{~d}$ after infection before $3 \mathrm{NP}$ intoxication (CNTF 30, $n=6$; CNTF 90, $n=5$ ) (Fig. 2E) did not alter the neuroprotective potential of the $C N T F$ treatment, despite a significant time-dependent increase in lesion volume observed in the noninfected striatum (factorial ANOVA; $p<0.01$ ), a phenomenon related to the age dependency of the toxicity of 3NP (Brouillet et al., 1993). Three months after administration of AdRSV.CNTF, PCR analysis demonstrated the presence of NGFp-CNTF transgene mRNA in two different AdRSV.CNTFinjected animals, in the infected (ipsilateral) striatum but not in the noninfected (contralateral) striatum (Fig. 3). 

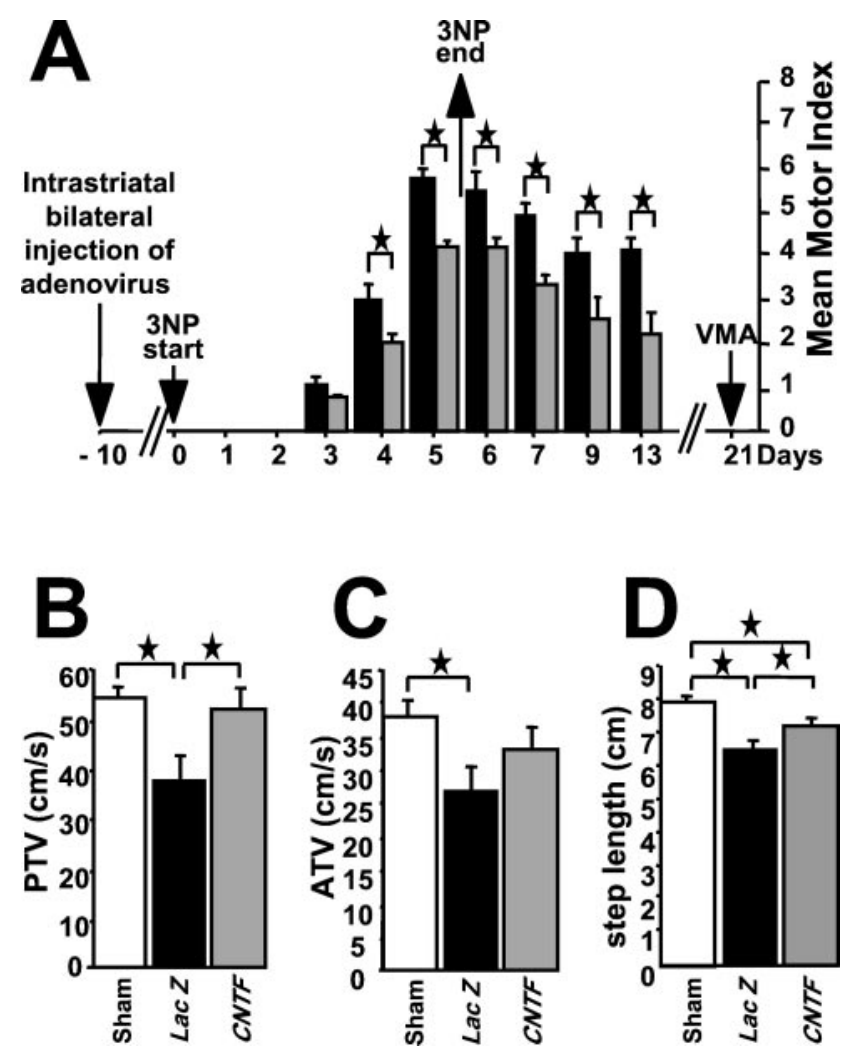

Figure 4. Experimental protocol $(A)$ in experiment 2 and long-term behavioral effects of the AdRSV.CNTF bilateral striatal injection on the parameters quantifying motor abnormalities $(A$, motor index) and motor performances ( $B$, PTV; $C$, ATV; $D$, step length) in the three different experimental groups. In $A$, note that from day 4 of the $3 \mathrm{NP}$ intoxication, animals injected with the AdRSV.CNTF adenovirus (gray bars) had a better motor index score than the animals injected with the AdRSV.LacZ adenovirus (black bars) $(\star p<0.05$; Mann-Whitney $U$ test). The beneficial effects of CNTF were different according to the parameters of motor performances. The mean value obtained for PTV (index of bradykinesia, $B$ ), was significantly higher in the CNTF group than in the LacZ group and was not different from that obtained with the sham group. For the mean values obtained for ATV (index of general activity, $C$ ), there was no significant difference between either the $C N T F$ and the sham groups or the LacZ and the CNTF groups, but there was a significant difference between the sham and the LacZ group. Finally, for the mean values obtained for step length $(D)$, there was no significant difference between the LacZ and the CNTF groups, but both groups were significantly different from the sham group $(\star p<0.05 ; \star \star p<0.0001$; ANOVA).

\section{Spatial features of the neuroprotective effects provided by AdRSV.CNTF (experiment 2)}

In animals challenged with a $5 \mathrm{~d}$ 3NP intoxication starting $10 \mathrm{~d}$ after bilateral administration of adenovirus vectors, the neuroprotective potential of $A d R S V . C N T F$ was studied using two different but complementary approaches: an in vivo qualitative (Fig. $4 A$ ) and quantitative (Fig. $4 B-D$ ) assessment of $3 \mathrm{NP}$-induced motor deficits, followed by postmortem stereological neuron counts in the striatum, the pallidum, and the medial agranular cortex of the same animals (Fig. 5).

As shown in Figure $4 A$, the first motor symptoms (lower limb intermittent dystonia) were observed in both experimental groups (animals treated with either AdRSV.LacZ or AdRSV.CNTF) on the third day of treatment. The motor symptoms increased in severity with time in both groups until the pumps were removed at day 5. At this time point, as well as on day 4, CNTF-treated animals $(n=8)$ were significantly less affected by the toxic

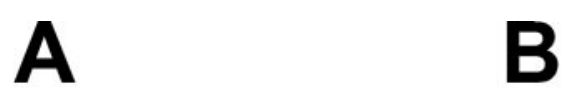

\section{medial agranular cortex}
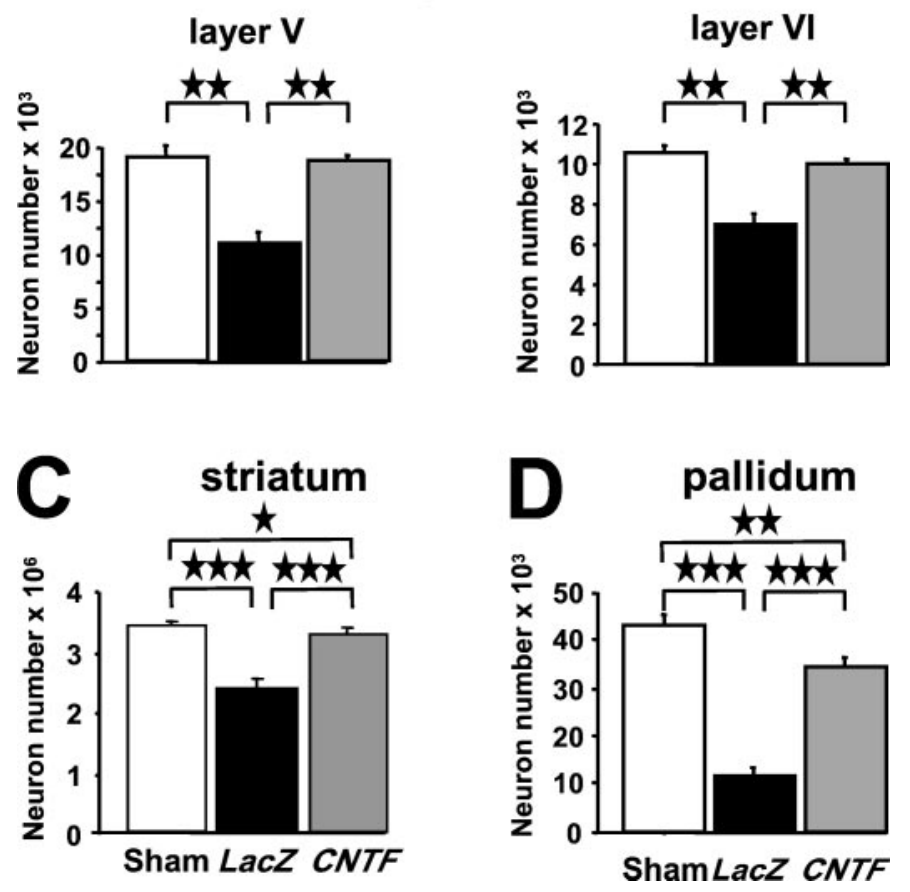

Figure 5. Protection of the neurons of the corticostriatopallidal pathway induced by a bilateral injection of AdRSV.CNTF adenovirus into the striatum (experiment 2). Stereological estimation of the number of neurons immunoreactive for NeuN in layers $\mathrm{V}$ and $\mathrm{VI}$ of the right medial agranular cortex $(A, B)$, in the striatum $(C)$, and in the pallidum $(D)$ shows that the protection of the striatal neurons is associated with a statistically significant protection of the cortical and pallidal neurons $(\star \star \star p<0.0001 ; \star \star p<0.002 ; \star p<0.02$; ANOVA).

treatment than the LacZ controls $(n=8)$ ( $p<0.05$; MannWhitney $U$ test). All animals progressively recovered after pump removal, but the $C N T F$-treated animals exhibited a lower motor score than the $L a c Z$ controls at all time points studied ( $p<0.05$; Mann-Whitney $U$ test). No significant difference in weight loss, recorded daily from day 1 to day 13, was observed between the two groups.

After a 2 week resting period, the motor performances of the three groups of animals (i.e., sham-operated animals, $A d R S V . C N T F$-injected animals, or AdRSV.LacZ-injected and $3 \mathrm{NP}$-intoxicated animals) were quantified in the elevated board test (Fig. $4 B-D)$. LacZ-injected animals $(n=8)$ displayed a significant decrease in PTV (an index of bradykinesia), a significant decrease in ATV (an index correlated with general locomotor activity), and a significant decrease in step length (resulting from severe bilateral leg dystonia) compared with the shamoperated animals $(n=9)$ ( $p<0.01$ vs sham animals). However, animals belonging to the AdRSV.CNTF group exhibited PTV values (Fig. 4B) and ATV values (Fig. $4 C$ ) that were not significantly different from sham performances. The average step length (Fig. 4D) was partially but significantly improved by $C N T F$ treatment (ANOVA; $p<0.05$; CNTF-treated vs either LacZ-treated or sham-operated animals).

Stereological neuronal cell counts (Table 2; Fig. 5) performed in the striatum and in regions containing afferent neurons to the striatum [layers $\mathrm{V}$ and VI of the rostral portion of the medial 


\begin{tabular}{|c|c|c|c|c|}
\hline Groups & Striatum & Pallidum & Cortical layer V & Cortical layer VI \\
\hline CNTF & $3,203,827 \pm 123,765^{* * * *}$ & $33,949 \pm 2803^{*, * *}$ & $17,622 \pm 782^{*}$ & $10,019 \pm 443^{*}$ \\
\hline Sham & $3,474,649 \pm 76,749$ & $45,824 \pm 3844$ & $18,869 \pm 967$ & $11,077 \pm 559$ \\
\hline LacZ & $2,366,442 \pm 134,448^{* *}$ & $12,668 \pm 1689^{* *}$ & $11,587 \pm 829^{* *}$ & $7,109 \pm 442^{* *}$ \\
\hline
\end{tabular}

${ }^{*} p<0.0001$ versus LacZ; ${ }^{*} p<0.002$ versus sham (factorial ANOVA; Sheffe's $F$ test).

agranular cortex, or efferent striatal target cells (globus pallidus)] (Reep et al., 1987; Berendse et al., 1992) were quite consistent with the motor scores. In all considered areas of the LacZinfected animals, the number of NeuN-immunoreactive neurons was significantly smaller than in sham animals, with decreases of 32,75 , and $39-36 \%$ observed in the striatum $(p<0.0001$; factorial ANOVA), the pallidum ( $p<0.0001)$, and layers V and VI of the cortex $(p<0.002)$, respectively. In the AdRSV.CNTF-injected animals, the numbers of NeuN-immunoreactive neurons in cortical layers V and VI were not significantly different from the numbers observed in sham animals, but they were barely reduced in the striatum ( $p<0.02$; factorial ANOVA) and significantly lower in the pallidum $(p<0.002)$. The neuroprotection induced by $C N T F$ was therefore complete in both cortical layers (99-96\% of sham values), whereas only partial neuroprotection was observed in the striatum (82\%; $p<0.02$ vs sham) and the pallidum $(70 \% ; p<$ 0.01 vs sham; factorial ANOVA; Scheffe's $F$ test).

As shown in Figure 6, total numbers of neurons in the striatum correlated linearly with total neuron numbers in the pallidum (Fig. 6A) $\left(r^{2}=0.51 ; p<0.0001\right)$ and in the rostral portion of medial agranular cortex layer V (Fig. $6 B)\left(r^{2}=0.57 ; p<0.0001\right)$ and layer VI (Fig. $6 C)\left(r^{2}=0.67 ; p<0.0001\right)$. Despite some intragroup heterogeneity, the LacZ-treated animals $(n=8)$ as a group were completely segregated from the two other experimental groups and the $C N T F$-treated animals $(n=8)$ were in the lower range of sham-operated animals $(n=9)$.

To examine further the correlation between behavioral and histological results, individual peak tangential velocities were plotted against total striatal neuron numbers. As shown in Figure 7 , there was a significant positive linear correlation between these two parameters $\left(r^{2}=0.547 ; p<0.003\right)$. Again, the group of rats infected with the AdRSV.LacZ vector $(n=8)$ was clearly separated from the sham-operated control animals $(n=9)$, whereas AdRSV.CNTF-treated rats $(n=8)$ were distributed in the lower normal range. The same positive correlation was found between striatal neuron numbers and ATV performance or step size (data not shown).

\section{DISCUSSION}

The present study shows that one single-site striatal injection of an adenovirus recombinant for $C N T F$ protects not only striatal neurons from degeneration but also cortical and pallidal neurons in a rat model of progressive striatal degeneration. These neuroprotective effects were associated with the persistence of transgene expression in the injected striatum up to 3 months after infection and correlated with a reduction of $3 \mathrm{NP}$-induced behavioral deficits. Together with previous data indicating the lack of adverse effects using the same adenoviral vector at similar low titers (Lisovoski et al., 1997), the current results show the therapeutic effect of adenovirus-mediated CNTF expression in the brain.

\section{Long-lasting transgene expression}

One of the issues addressed in the present study was the demonstration of a long-lasting efficacy of cell infection with adenovirusmediated gene transfer. Because adenovirus vectors do not integrate their genome into that of the transduced cells but rather express it as an episome, it is generally believed that such gene expression is only transient (Lee et al., 1993; Kozarsky et al., 1994). Although this has been demonstrated clearly for cells that divide after infection (Boviatsis et al., 1994), it is less well established for nondividing cells such as neurons. To address the issue of the duration of transgene expression in neurons, most authors have relied on histochemical and/or immunocytochemical detection of the transgene product (Akli et al., 1993; Davidson et al., 1993; Le Gal La Salle et al., 1993; Bennett et al., 1994; Ghodsi et al., 1998; Bohn et al., 1999). Although the main objective was to examine the neuroprotective effects of adenoviruses delivering CNTF on maintaining the anatomical integrity of the structures compromised in this animal model, we also measured behavioral effects. The results clearly demonstrate neuroprotective effects in terms of stereological counts and in some behavioral parameters. The neuroprotection correlates with complete behavioral sparing in one of the parameters tested (peak tangential velocity) and with a partial sparing in step length, two parameters particularly sensitive to 3NP intoxication (Ouary et al., 2000). The other kinetic parameter, ATV, while being significantly altered by the $3 \mathrm{NP}$ lesion, does not appear to be as restored by CNTF. The reason for this difference is unclear and merits further investigation.

The use of stereological cell counts and behavioral studies demonstrates the neuroprotective efficacy of the transgene product up to 3 months after intrastriatal delivery of the viral vector. Although this interval is still too short for a therapeutic use in patients, it does indicate a protective effect of the transgene over 3 months. It also compares favorably with the available data concerning other gene therapy delivery systems for a trophic factor to the brain, such as adenoassociated virus vectors (Bosch et al., 2000; Dhillon et al., 2000; Kirik et al., 2000), lentivirus vectors (Kordower et al., 2000; Consiglio et al., 2001), and encapsulated engineered cells (Mittoux et al., 2000) that, up to now, have shown relatively short survival times, requiring frequent exchange of the device (Bachoud-Lévi et al., 2000).

\section{Retrograde infection of projecting neurons and anterograde transport of the transgene product: two properties of adenovirus vectors}

In the present study, we demonstrate an effect of CNTF well beyond the transfected striatal area. These results can be explained by a combination of intrinsic properties of adenoviral vectors and CNTF on the one hand and the anatomical organization of the basal ganglia on the other. At the injection site (Fig. 8), mostly neurons but also glial cells are transfected (Peltekian et al., 1997). Moreover, it is known that adenoviral vectors are taken 

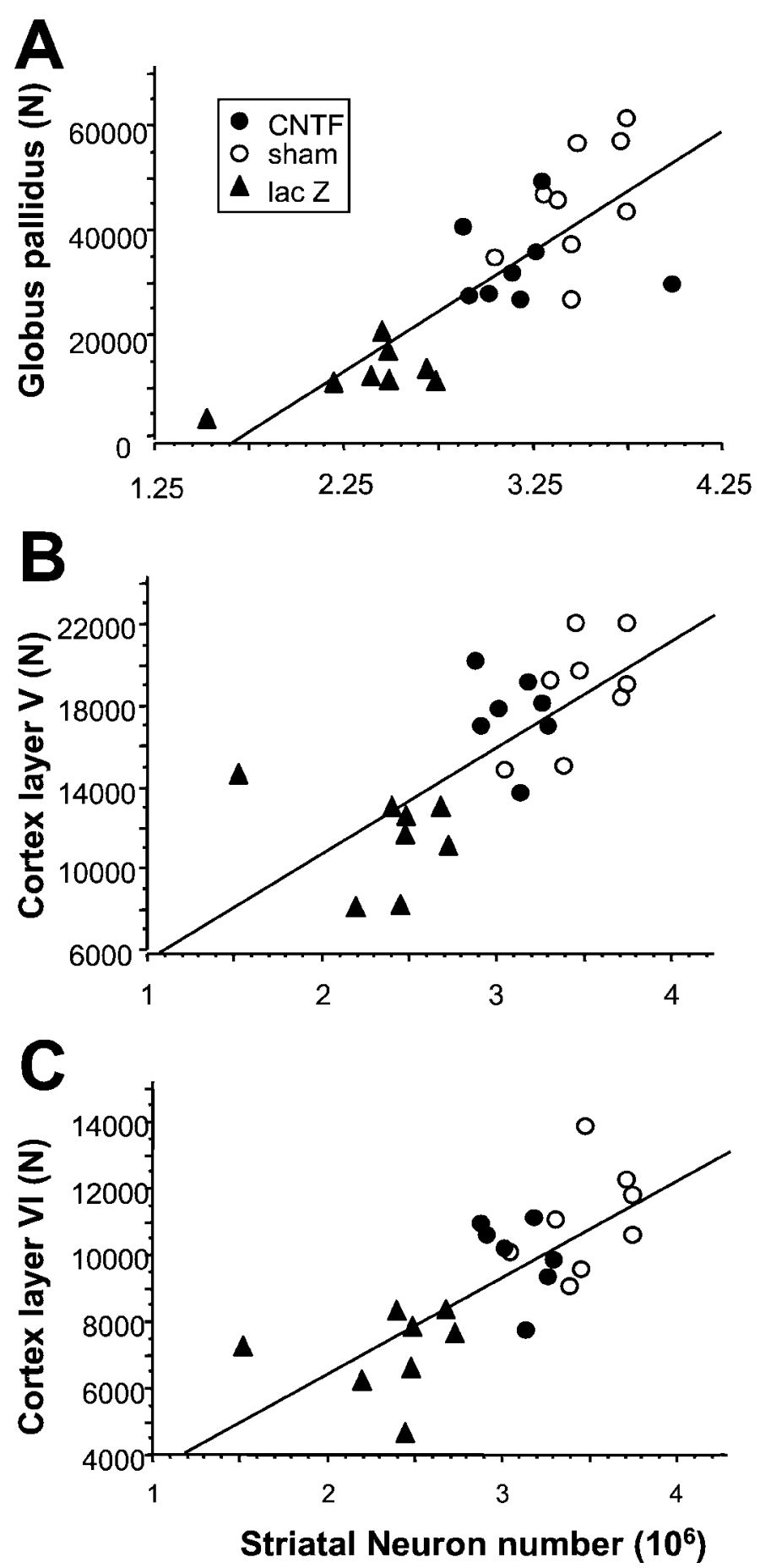

Figure 6. Linear correlations between the number of striatal NeuNimmunoreactive neurons and the number of neurons in the pallidum $(A)$ and in layers $\mathrm{V}(B)$ or VI $(C)$ of the medial agranular frontal cortex.(A, $\left.r^{2}=0.51, p<0.0001 ; B, r^{2}=0.57, p<0.0001 ; C, r^{2}=0.67, p<0.0001\right)$.

up by axon terminals located in the infected area (in the present case, the striatum) and are retrogradely transported to neuronal cell bodies (Fig. 8) in the cerebral cortex, the substantia nigra pars compacta, and other striatal afferent structures (Akli et al., 1993; Bajocchi et al., 1993; Ridoux et al., 1994; Kuo et al., 1995). The adenoviral vector then accumulates in the infected cell bodies and it expresses the transgene, which is in turn anterogradely transported to axonal terminals and released (Lisovoski et al., 1997;

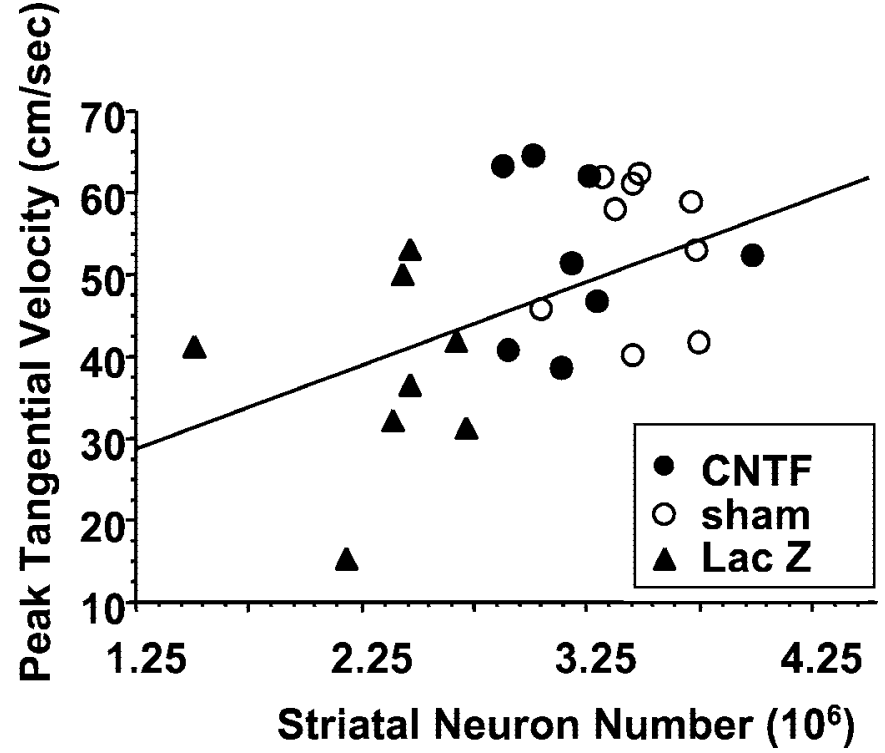

Figure 7. Linear correlation between the number of striatal NeuNimmunoreactive neurons and the PTV of animals belonging to the AdRSV.CNTF, AdRSV.LacZ, and sham groups (experiment 2). $r^{2}=$ $0.547 ; p<0.003$.

Terashima et al., 1997). In our case, the main area concerned by the release of anterogradely transported transgene product is the globus pallidus, which receives the bulk of its afferents from the striatum. In addition, an intrastriatal release of transgenic CNTF originating from the retrogradely transfected cortical areas and substantia nigra may contribute to the neuroprotective effects observed here.

Thus, local and distal expression of the transgene serves to furnish CNTF to the striatum as well as to its afferent cortical areas and to the efferent globus pallidus. The transgenic CNTF released by the infected cells acts not only on these cells themselves (Hudgins and Levison, 1998) but also on the neuronal and glial cells located within its diffusion zone (Baumgartner and Shine, 1997), a process that further increases the sphere of the neuroprotective effects of CNTF. CNTF induces astrocyte differentiation and the upregulation of astrocyte CNTF synthesis, thereby providing additional paracrine support to neurons (Clatterbuck et al., 1996; Lisovoski et al., 1997). Because astrocytes are functionally as well as anatomically connected (Kim et al., 1994), infection of local astrocytes by the AdRSV.CNTF vector may in turn stimulate the subsequent synthesis and release of endogenous CNTF by noninfected astrocytes located at a distance from the vector injection.

Finally, the anatomical organization of the corticostriatopallidal circuitry itself can influence the dispersion of viral particles, because the transfection follows the connectivity. For example, because corticostriatal input fibers are organized in a patchy way within the striatum (Graybiel et al., 1991), the striatal volume covered by the adenovirus injection can contain parts of one or several of these patches, which will determine the number and the volume of the cortical areas retrogradely transfected. However, because of the convergence of corticostriatal inputs (Kincaid et al., 1998), a large number of cortical neurons are potentially able to be retrogradely transfected from a very restricted adenovirus injection. These retrogradely transfected neurons will serve as new sources of transgenic CNTF. In contrast, the high degree of convergence and the spatial organization of the striatopallidal 


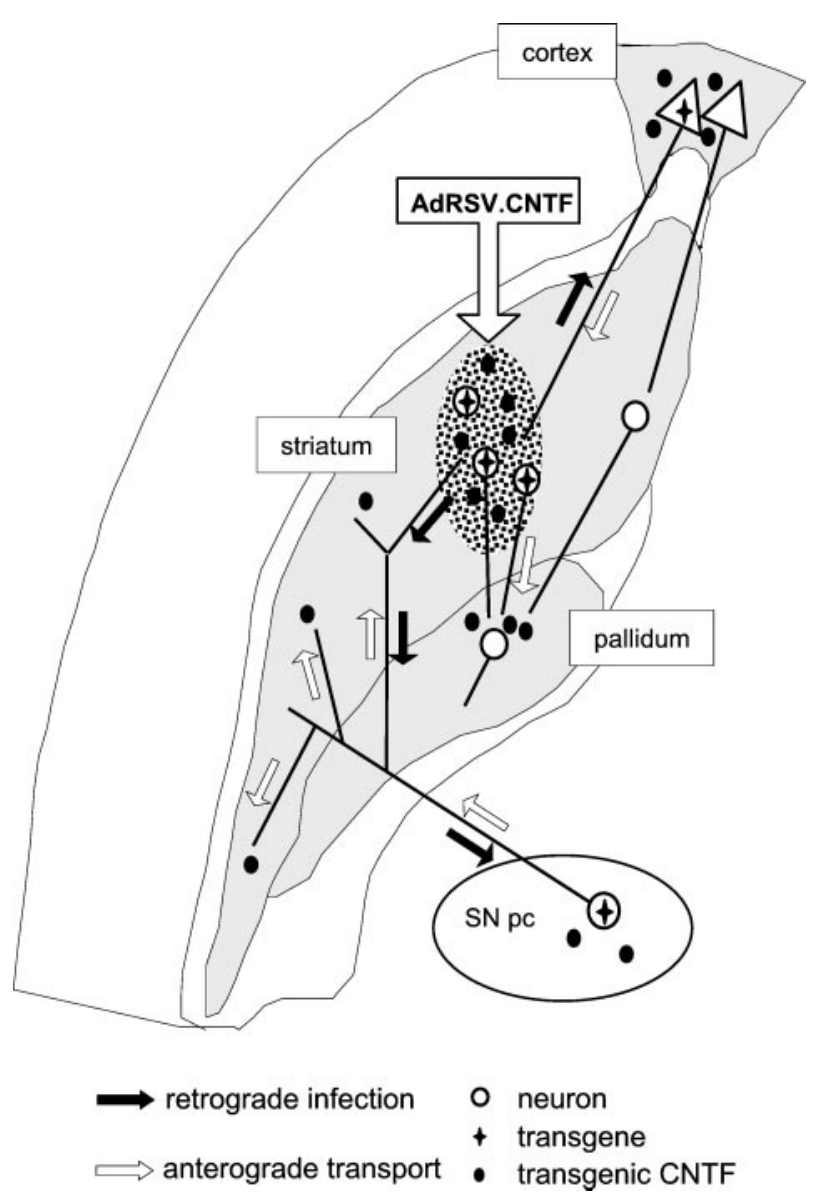

Figure 8. Hypothetical mechanisms underlying CNTF-induced neuroprotection in the corticostriatopallidal neuronal circuitry. In the infected striatal area (dotted region) primarily neurons but also glial cells and axon terminals are transfected. Adenoviral particles taken up by axon terminals located in the infected area are retrogradely transported to the corresponding cell bodies in the cerebral cortex and the substantia nigra pars compacta $(S N p c)$. Transgenes accumulated in the infected cell bodies express the transgenic CNTF, which in turn can be released locally or anterogradely transported down to axonal terminals and released. Through a combination of direct infection and both retrograde and anterograde intra-axonal transport of transgene and transgene product, respectively, the neuroprotective agent CNTF can be released and act within the striatum as well as in its main afferent (cortex, $S N p c$ ) or efferent (pallidum) cerebral structures (gray shaded areas).

connections (Yelnik et al., 1996) would confine the release of transgenic CNTF only to a restricted part of the globus pallidus. These anatomical organizations may account for the differences in the neuroprotection observed in cortical layers and in the pallidum.

Together, our data strongly support the notion that the initial spatial restriction of the adenoviral infection around the site of administration may not be a major problem in neuroprotective therapeutic trials for HD.

\section{Toward a clinical use of adenovirus-mediated CNTF neuroprotection}

The present study demonstrates that a single administration of noncytotoxic amounts of adenoviral particles can be sufficient to ensure widespread and long-lasting delivery of a therapeutic transgene product. These results underscore the potential clinical use of adenoviral vectors as a means to transfer genes into patients with HD. Previous studies have indicated the potential neuroprotective set of CNTF in the striatum using either implanted minipumps (Anderson et al., 1996) or encapsulated engineered cells for delivery (Emerich et al., 1996; Mittoux et al., 2000). The present study extends these observations by showing that CNTF-expressing adenovirus vectors can neuroprotect neurons located upstream and downstream from the striatum, and in particular, the corticostriatopallidal pathway, a neuronal circuit severely affected in HD. In addition to the potential interest of an extrastriatal neuroprotection in HD, it is worth noting that this may also benefit other pathologies that affect both striatal and nigral neurons, such as multiple system atrophy or Steele-Richardson syndrome.

\section{REFERENCES}

Akli S, Caillaud C, Vigne E, Stratford-Perricaudet LD, Poenaru L, Perricaudet M, Kahn A, Peschanski MR (1993) Transfer of a foreign gene into the brain using adenovirus vectors. Nat Genet 3:224-228.

Anderson KD, Panayatatos N, Corcoran TL, Lindsay RM, Wiegand S (1996) Ciliary neurotrophic factor protects striatal outputs neurons in an animal model of Huntington disease. Proc Natl Acad Sci USA 93:7346-7351.

Bachoud-Lévi AC, Déglon N, Nguyen JP, Bloch J, Bourdet C, Winkel L, Rémy P, Goddard M, Lefaucheur JP, Brugières P, Baudic S, Cesaro P, Peschanski M, Aebischer P (2000) Neuroprotective gene therapy for Huntington's disease using a polymer encapsulated BHK cell line engineered to secrete human CNTF. Hum Gene Ther 11:1723-1729.

Bajocchi G, Feldman SH, Crystal RG, Mastrangeli A (1993) Direct in vivo gene transfer to ependymal cells in the central nervous system using recombinant adenovirus vectors. Nat Genet 3:229-234.

Baumgartner BJ, Shine HD (1997) Targeted transduction of CNS neurons with adenoviral vectors carrying neurotrophic factor genes confer neuroprotection that exceeds the transduced population. J Neurosci 17:6505-6511.

Beal MF, Brouillet E, Jenkins BG, Ferrante RJ, Kowall NW, Miller JM, Storey E, Srivastava R, Rosen BR, Hyman BT (1993) Neurochemical and histologic characterization of striatal excitotoxic lesions produced by the mitochondrial toxin 3-nitropropionic acid. J Neurosci 13:4181-4192.

Bennett J, Wilson J, Sun D, Forbes B, Maguire A (1994) Adenovirus vector-mediated in vivo gene transfer into adult murine retina. Invest Ophthalmol Vis Sci 35:2535-2542.

Berendse HW, Galis-de Graaf Y, Groenewegen HJ (1992) Topographical organization and relationship with ventral striatal compartments of prefrontal corticostriatal projections in the rat. J Comp Neurol 316:314-347.

Bohn MC, Choi-Lundberg DL, Davidson BL, Leranth C, Kozlowski DA, Smith JC, O'Banion MK, Redmond Jr DE (1999) Adenovirusmediated transgene expression in nonhuman primate brain. Hum Gene Ther 10:1175-1184.

Bosch A, Perret E, Desmaris N, Heard JM (2000) Long-term and significant correction of brain lesions in adult mucopolysaccharidosis type VII mice using recombinant AAV vectors. Mol Ther 1:63-70.

Boviatsis EJ, Chase M, Wei MX, Tamiya T, Hurford Jr RK, Kowall NW, Tepper RI, Breakefield XO, Chiocca EA (1994) Gene transfer into experimental brain tumors mediated by adenovirus, herpes simplex virus and retrovirus vectors. Hum Gene Ther 5:183-191.

Brouillet E, Jenkins BG, Hyman BT, Ferrante RJ, Kowall NW, Srivastava R, Samanta Roy D, Rosen BR, Beal MF (1993) Age-dependent vulnerability of the striatum to the mitochondrial toxin 3-nitropropionic acid. J Neurochem 60:356-359.

Byrnes AP, Rusby JE, Wood MJ, Charlton HM (1995) Adenovirus gene transfer causes inflammation in the brain. Neuroscience 66:1015-1024.

Caillaud C, Akli S, Vigne E, Koulakoff A, Perricaudet M, Poenaru L, Kahn A, Berwald-Netter Y (1993) Adenoviral vector as a gene delivery system into cultured rat neuronal and glial cells. Eur J Neurosci 5:1287-1291.

Clatterbuck RE, Price DL, Koliatsos VE (1996) Ciliary neurotrophic factor stimulates the expression of glial fibrillary acidic protein by brain astrocytes in vivo. J Comp Neurol 369:543-551.

Consiglio A, Quattrini A, Martino S, Bensadoun JC, Dolcetta D, Trojani A, Benaglia G, Marchesini S, Cestari V, Oliverio A, Bordignon C, Naldini L (2001) In vivo gene therapy of metachromatic leukodystrophy by lentiviral vectors: correction of neuropathology and protection against learning impairments in affected mice. Nat Med 7:310-316.

Davidson BL, Allen ED, Kozarski KF, Wilson JM, Roessler JB (1993) A model system for in vivo gene transfer into the central nervous system using an adenoviral vector. Nat Genet 3:219-223.

Dhillon H, Ge Y, Minter RM, Prima V, Moldawer LL, Muzyczka N, Zolotukhin S, Kalra PS, Kalra SP (2000) Long-term differential modulation of genes encoding orexigenic and anorexigenic peptides by 
leptin delivered by rAAV vector in ob/ob mice. Relationship with body weight change. Regul Pept 92:97-105.

Emerich DF, Lindner MD, Winn SR, Chen EY, Frydel BR, Kordower JH (1996) Implants of encapsulated human CNTF-producing fibroblasts prevent behavioral deficits and striatal degeneration in a rodent model of Huntington's disease. J Neurosci 16:5168-5181.

Ghodsi A, Stein C, Derksen T, Yang G, Anderson RD, Davidson BL (1998) Extensive beta-glucuronidase activity in murine central nervous system after adenovirus-mediated gene transfer to brain. Hum Gene Ther 9:2331-2340.

Graybiel AM, Flaherty AW, Gimenez-Amaya JM (1991) Striosomes and matrisomes. In: The basal ganglia, Vol III (Bernardi G, Carpenter MB, di Chiara G, Morelli M, Stanzione P, eds), pp 3-12. New York: Plenum.

Guyot MC, Hantraye P, Dolan R, Palfi S, Mazière M, Brouillet E (1997) Quantifiable bradykinesia, gait abnormalities and Huntington diseaselike striatal lesions in rats chronically treated with 3-nitropropionic acid. Neuroscience 79:45-56.

Haase G, Pettmann B, Bordet T, Villa P, Vigne E, Schmalbruch H, Kahn A (1999) Therapeutic benefit of ciliary neurotrophic factor in progressive motor neuronopathy depends on the route of delivery. Ann Neurol 45:296-304.

Hudgins SN, Levison SW (1998) Ciliary neurotrophic factor stimulates astroglial hypertrophy in vivo and in vitro. Exp Neurol 150:171-182.

Kim WT, Rioult MG, Cornell-Bell AH (1994) Glutamate-induced calcium signaling in astrocytes. Glia 11:173-184.

Kincaid AE, Zheng T, Wilson CJ (1998) Connectivity and convergence of single corticostriatal axons. J Neurosci 18:4722-4731.

Kirik D, Rosenblad C, Björklund A, Mandel RJ (2000) Long-term rAAV-mediated gene transfer of GDNF in the rat Parkinson's model: intrastriatal but not intranigral transduction promotes functional regeneration in the lesioned nigrostriatal system. J Neurosci 20:4686-4700.

Kordower JH, Emborg ME, Bloch J, Ma SY, Chu Y, Leventhal L, McBride J, Chen EY, Palfi S, Roitberg BZ, Brown WD, Holden JE, Pyzalski R, Taylor MD, Carvey P, Ling Z, Trono D, Hantraye P, Déglon N, Aebischer P (2000) Neurodegeneration prevented by lentiviral vector delivery of GDNF in primate models of Parkinson's disease. Science 290:767-773.

Kozarsky KF, McKinley DR, Austin LL, Raper SE, Stratford-Perricaudet LD, Wilson JM (1994) In vivo correction of low density lipoprotein receptor deficiency in the Watanabe heritable hyperlipidemic rabbit with recombinant adenoviruses. J Biol Chem 269:13695-13702.

Kuo H, Ingram DK, Crystal RG, Mastrangeli A (1995) Retrograde transfer of replication deficient recombinant adenovirus vector in the central nervous system for tracing studies. Brain Res 705:31-38.

Lee SW, Trapnell BC, Rade JJ, Virmani R, Dichek DA (1993) In vivo adenoviral vector-mediated gene transfer into balloon-injured rat carotid arteries. Circ Res 73:797-807.
Le Gal La Salle G, Robert JJ, Berrard S, Ridoux V, Stratford-Perricaudet LD, Perricaudet M, Mallet J (1993) An adenovirus vector for gene transfer into neurons and glia in the brain. Science 259:988-990.

Lisovoski F, Akli S, Peltekian E, Vigne E, Haase G, Perricaudet M, Dreyfus PA, Kahn A, Peschanski M (1997) Phenotypic alteration of astrocytes induced by ciliary neurotrophic factor in the intact adult brain, as revealed by adenovirus-mediated gene transfer. J Neurosci 17:7228-7236.

Mittoux V, Joseph JM, Condé F, Palfi S, Dautry C, Poyot T, Bloch J, Déglon N, Ouary S, Nimchinsky EA, Brouillet E, Hof PR, Peschanski M, Aebischer P, Hantraye P (2000) Restoration of cognitive and motor functions by ciliary neurotrophic factor in a primate model of Huntington's disease. Hum Gene Ther 11:1177-1187.

Ouary S, Bizat N, Altairac S, Ménetrat H, Mittoux V, Condé F, Hantraye P, Brouillet E (2000) Major strain differences in response to chronic systemic administration of the mitochondrial toxin 3-nitropropionic acid in rats: implications for neuroprotection studies. Neuroscience 97:521-530.

Paxinos G, Watson C (1986) The rat brain in stereotaxic coordinates, Ed 2. San Diego: Academic.

Peltekian E, Parrish E, Bouchard C, Peschanski M, Lisovoski F (1997) Adenovirus-mediated gene transfer to the brain: methodological assessment. J Neurosci Methods 71:77-84.

Reep RL, Corwin JV, Hashimoto A, Watson RT (1987) Efferent connections of the rostral portion of medial agranular cortex in rats. Brain Res Bull 19:203-221.

Ridoux V, Robert JJ, Zhang X, Perricaudet M, Mallet J, Le Gal La Salle $G$ (1994) Adenoviral vectors as functional retrograde neuronal tracers. Brain Res 648:171-175.

Saudou F, Finkbeiner S, Devys D, Greenberg ME (1998) Huntingtin acts in the nucleus to induce apoptosis but death does not correlate with the formation of intranuclear inclusions. Cell 95:55-66.

Schmued LC, Albertson C, Slikker Jr W (1997) Fluoro-Jade: a novel fluorochrome for the sensitive and reliable histochemical localization of neuronal degeneration. Brain Res 751:37-46.

Terashima T, Miwa A, Kanegae Y, Saito I, Okado H (1997) Retrograde and anterograde labeling of cerebellar afferent projection by the injection of recombinant adenoviral vectors into the mouse cerebellar cortex. Anat Embryol 196:363-382.

West MJ, Gundersen HJ (1990) Unbiased stereological estimation of the number of neurons in the human hippocampus. J Comp Neurol 296:1-22.

Yelnik J, François C, Percheron G, Tandé D (1996) A spatial and quantitative study of the striatopallidal connection in the monkey. NeuroReport 7:985-988. 